\title{
Apprentissage de la langue et comparatisme culturel en Hollande : le métier de maître de langue (XVII ${ }^{\mathrm{e}}$-XIX ${ }^{\mathrm{e}}$ siècle)
}

Marie-Christine Kok Escalle, Université Ultrecht, Madeleine Van StrienChardonneau et Universiteit Leiden

\section{OpenEdition}

\section{Journals}

Édition électronique

URL : https://journals.openedition.org/dhfles/1745

DOI : $10.4000 /$ dhfles. 1745

ISSN : 2221-4038

Éditeur

Société Internationale pour l'Histoire du Français Langue Étrangère ou Seconde

Édition imprimée

Date de publication : 1 juin 2005

ISSN : 0992-7654

Référence électronique

Marie-Christine Kok Escalle, Université Ultrecht, Madeleine Van Strien-Chardonneau et Universiteit Leiden, « Apprentissage de la langue et comparatisme culturel en Hollande : le métier de maître de langue (XVIIe-XIX siècle) », Documents pour l'histoire du français langue étrangère ou seconde [En ligne], 33/34 | 2005, mis en ligne le 01 janvier 2012, consulté le 27 mai 2021. URL : http:// journals.openedition.org/dhfles/1745; DOI : https://doi.org/10.4000/dhfles.1745

Ce document a été généré automatiquement le 27 mai 2021.

(c) SIHFLES 


\title{
Apprentissage de la langue et comparatisme culturel en Hollande : le métier de maître de langue (XVII ${ }^{\mathrm{e}}-\mathrm{XIX}{ }^{\mathrm{e}}$ siècle)
}

\author{
Marie-Christine Kok Escalle, Université Ultrecht, Madeleine Van Strien- \\ Chardonneau et Universiteit Leiden
}

Selon G.G. Baardman ${ }^{1}$, l'enseignement des langues modernes aux Pays-Bas présente, à la fin du XIXe siècle, dans sa diversité, peu de différence avec le XVIe siècle: s'y opposent la méthode directe et la méthode grammaticale, que celle-ci soit inductive ou déductive. Du XVIe au XVIIIe siècle, le français y est enseigné dans des écoles dites françaises (Franse scholen) par des maîtres et maîtresses ; ceux-ci, maîtres d'école, sont aussi maîtres de langue française, langue qu'ils parlent, écrivent et utilisent comme langue d'enseignement. Les écoles latines en revanche dispensent un enseignement de langues classiques. Dans les milieux aristocratiques, l'enseignement du français passe par la pratique éducative confiée aux gouvernantes et précepteurs. Avec le développement de l'éducation scolaire au XIXe siècle, l'enseignement des langues étrangères va entrer dans les structures institutionnelles de l'enseignement primaire puis secondaire et enfin universitaire. Et si c'est seulement au $\mathrm{XX}^{\mathrm{e}}$ siècle que l'on se préoccupe de mettre en place concrètement les structures de formation pédagogique des maîtres ${ }^{2}$, ceux-ci n'en ont pas moins, au long des siècles, livré leurs réflexions sur leur pratique, celle d'un véritable métier de maître de langue.

\section{Institutionnalisation tardive de l'enseignement du français au niveau du secondaire et dans le supérieur}

On assiste en effet aux Pays-Bas à une institutionnalisation fort tardive et lacunaire du métier de professeur de langues ${ }^{3}$, de son ancrage dans une formation universitaire et de sa reconnaissance par la délivrance de diplômes. 
3 La loi de 1806 sur l'enseignement primaire stipule dans son article I que les écoles primaires «enseignent les bases du savoir et de la civilisation à savoir la lecture, l'écriture, le calcul, la langue néerlandaise et d'autres matières auxiliaires comme le français et autres langues modernes ou savantes, l'histoire et la géographie ». Le maître d'école primaire doit pouvoir parler suffisamment français - et cette compétence est vérifiée avant toute nomination - pour pouvoir l'enseigner à ses élèves, ce qui reste certainement très théorique et loin de la réalité mais répond à une volonté publique d'éducation de la jeunesse conforme à l'idéal des Lumières. La loi de 1857 confirme cette situation en précisant que les bases de l'enseignement des langues vivantes seront données dans le primaire et approfondies dans le secondaire dont on prépare la création. Elle instaure en outre un examen à passer pour obtenir le certificat d'aptitude à l'enseignement primaire, qui comprend une épreuve de langues vivantes étrangères (prononciation, élocution, traduction, bases de la grammaire, courants littéraires, savoir parler et écrire).

4 Sans qu'il y ait de formation professionnelle on attend donc de l'instituteur, dès le début du XIX siècle, qu'il ait une connaissance de langue et culture étrangère et en l'occurrence du français. Apprendre en langue étrangère est considéré comme donnant une ouverture linguistique et culturelle et comme enrichissant la connaissance et compréhension de sa propre langue et culture. Depuis le début du XVI ${ }^{\mathrm{e}}$ siècle d'ailleurs, le français est dans les Provinces-Unies, langue d'éducation et de culture.

5 A côté de l'école primaire il y a l'école latine, athénée ou gymnase, où l'on enseigne les langues classiques. Quelques essais ont été faits pour introduire dans certains gymnases d'avant-garde des grandes villes du pays (La Haye, Utrecht, Nimègue, Hardewijk), un enseignement de français dès 1838 mais les langues modernes disparaîtront du gymnase lorsqu'elles apparaîtront dans les HBS.

6 La loi de 1863 (Thorbecke) pour le secondaire crée les écoles bourgeoises et les écoles bourgeoises supérieures (HBS), l'accès y étant soumis à un examen d'entrée qui jusqu'en 1920 comporte une épreuve de français; dans ces HBS il est prescrit un enseignement de trois langues modernes, le français, l'anglais et l'allemand (dans cet ordre). On doit donc faire appel à de nombreux étrangers pour assurer ces enseignements avant que ne soient créés les actes de qualification pour l'enseignement secondaire (MO Akte) par la loi de 1879. L'association des professeurs de langues vivantes Levende Talen sera créée en 1911 et son organe de presse, Berichten en mededelingen en 1914.

7 La loi de 1876 sur l'enseignement supérieur prévoit une chaire pour trois langues modernes dans au moins une université du pays, mais l'application de la loi sera lente. Groningue choisit de créer les chaires, pensant ainsi assurer la survie de son établissement universitaire; les nominations interviendront en 1881 pour la chaire d'allemand (Sijmons), en 1884 pour la chaire de français (van Hamel) et en 1885 pour la chaire d'anglais (Beekering Vinckers) 4 .

8 Si l'idée d'offrir une formation aux professeurs de langues du secondaire justifie la loi qui crée ces chaires, en réalité l'université offrira une formation philologique et linguistique fondée sur une approche historique, mais pas de didactique ni de pédagogie. En outre, il faudra attendre 1912 pour qu'une chaire de français soit pourvue à l'université d'Amsterdam et 1921 pour que le statut académique fixe les conditions d'attribution d'un diplôme, créé en 1919 pour sanctionner les études de langues vivantes étrangères à l'université. 
Les lois de 1863 et de 1876 mettent en place une institutionnalisation de l'enseignement des langues vivantes étrangères dont la langue française, mais la professionnalisation reste absente et la disciplinarisation s'opère très lentement. En effet, les examens mis en place en 1879 sanctionneront un savoir, une aptitude à parler et écrire, à traduire, mais pas un savoir-faire professionnel (MO Akte A pour la langue et B pour la langue et la littérature). Ensuite les objectifs universitaires de formation des maîtres (cours de didactique) ne sont pas suivis ${ }^{5}$, ceux de formation à une discipline se limitent à la philologie historique ${ }^{6}$. Enfin créer la chaire de langue étrangères à l'université de Groningue, c'est affirmer le changement de statut de la langue moderne qui d'une compétence de savoir faire devient une formation scientifique et disciplinaire; pourtant entre 1881 et 1884 on cherchera en vain un titulaire qui pourrait occuper en même temps la chaire de français et celle d'anglais et il reste très difficile de faire accepter les langues étrangères comme égales aux langues anciennes et à la langue maternelle, dans le complexe des études universitaires (Sijmons, 434). Cependant, même si la reconnaissance institutionnelle de la profession et de la discipline est fort tardive, l'enseignement du français aux Pays-Bas est dispensé depuis le début du XVIe siècle par des hommes de métier.

\section{Longue tradition du métier de maître de langues}

10 Le maitre de langues est un praticien plus soucieux d'efficacité que de théorie et qui, riche de son expérience, propose des modèles de pratiques à travers ses ouvrages. Instructeur et éducateur, sa fonction dépasse la discipline. Mais il a une idée de son métier et tente sinon de théoriser sa pratique, du moins de l'expliquer et de la justifier dans les préfaces, les avis aux lecteurs, mais aussi dans les dialogues destinés à la conversation qui mettent souvent en scène maîtres et élèves.

11 Notre corpus pour des raisons d'ordre pratique se limite à quelques noms parmi le très grand nombre de ceux qui ont exercé le métier de maître de langues en Hollande du $\mathrm{XVII}^{\mathrm{e}}$ au XIX ${ }^{\mathrm{e}}$ siècle ou dont les méthodes ont été longuement utilisées. Si le choix opéré n'échappe pas complètement à l'arbitraire, il tient cependant compte d'une part, des maîtres dont les méthodes ont connu un succès certain et durable, d'autre part, des deux grands types d'enseignement du français, à savoir l'enseignement privé touchant des individus d'âge varié et celui dispensé aux enfants dans les écoles dites françaises. J.N. Parival, B. Piélat, C. Mauger, pour le XVII ${ }^{e}$ siècle sont des maîtres de langue dont la pratique et les méthodes concernent plutôt l'enseignement individuel comme c'est le cas aussi au XVIII pour l'abbé de Bellegarde et I. de Charrière, alors que P. Marin qui exerce aussi bien à titre privé qu'à titre de maître d'école produit, à l'usage des écoliers, des méthodes qui seront rééditées tout au long du siècle et reprises encore au XIX ${ }^{e}$. Quant à J. Ebrard du Casquet, Ch. Cazelles, D. Bomhoff, E. Peel, A.-N. Agron, P.J. Baudet, J.-J. Gilbert, Fournier, ces maîtres du XIX ${ }^{e}$ siècle rédigent leurs manuels à l'intention d'un public scolaire.

\section{2a. Le métier de maître de langues}

12 Le maître est-il indispensable? C'est une question qui se pose parfois. Il arrive que les ouvrages des maîtres soient présentés comme pouvant remplacer ceux-ci : ainsi l'Avertissement du libraire vante l'Anti-Grammaire de Piélat comme la méthode 
incontournable pour apprendre le français et fait le procès des maîtres de langues. Piélat lui-même, dans ses Letres nouveles et curieuses (Paris 1677), oppose l'enseignement déficient de l'école, donc des maîtres, à l'excellence de sa méthode :

[...] il est impossible d'aprendre bien la langue françoise autrement que par une seule Methode. Vous sçavés que vous avés esté plusieurs années dans une des meilleures Escoles Françoises de toute la Hollande; Et ensuite autant que vous avés esté fachée de voir les fautes que vous commetiés depuis si long-temps, autant avés vous esté satisfaite de vous en corriger en peu de jours. [...] Maintenant vous pouvés estre assurée qu'il n'a point de Maistre de langues qui vous surpasse (153-154).

Ou bien, il s'agit d'ouvrages s'adressant à des élèves déjà formés, comme au XIX ${ }^{\mathrm{e}}$ siècle ceux de Baudet ${ }^{8}$, dont les volumes VII et VIII des Exercices s'adressent plus particulièrement à ceux qui ont quitté l'école et qui « lors même qu'ils seront entrés dans le monde » (Exercices VII [1842] $4^{\mathrm{e}}$ éd. 1860, 3-4) pourront continuer l'étude. Cependant le maître est lié à l'apprentissage de la langue, c'est du mauvais maître, du " gaste-métier » dont il faut se méfier et la qualité du maître est présentée comme primordiale.

14 Mais qu'est-ce qu'un bon maître? Dans ses Dialogues ${ }^{9}$, Parival met en valeur l'expérience pratique et la qualité de la méthode. Pour Piélat comme pour Mauger, on peut déduire d'après les Avertissements et Avis au lecteur surtout ce que le bon maître ne doit pas être : un pédant à la tête farcie de règles grammaticales ou en d'autres termes un théoricien de la langue peu intéressé au fond par les apprenants, un de ces " grammairiens [qui] accablent les esprits de leurs disciples, avec le plus grand nombre de leurs préceptes, exceptions, \& sous exceptions » (Piélat, L'Antigrammaire, Sommaire).

Plutôt que de théorie, le bon maître doit donc être soucieux de l'efficacité de sa pratique. Et cette efficacité repose d'abord bien entendu sur une bonne connaissance de la langue enseignée et en particulier une bonne prononciation : à ce propos, on peut signaler que la qualité de locuteur natif, importante certes, n'est pas forcément garante de la qualité du maître ${ }^{10}$, comme on peut le déduire de ce passage emprunté à Mauger :

L'on m'a dit de beaucoup de François, qui parloyent fort mal quand ils sortirent de France, étant ignorants, \& nés dans des provinces où le langage est fort rude, qu'ils ont appris à parler bon françois à La Haye (239) ${ }^{11}$. Le bon maitre doit être aussi conscient des besoins de ses élèves. C'est ainsi que Piélat, s'adressant " aux lecteurs de la clavicule de la langue française ${ }^{12}$, précise que son ouvrage est destiné à deux catégories d'apprenants, d'une part ceux qui veulent pouvoir lire et converser et qui ne sont donc intéressés que par la pratique de la langue, d'autre part, ceux qui ne sont pas très doués pour les études (Letres nouveles \& curieuses, 265-266). Et si les ouvrages de Marin ${ }^{13}$ ont connu un très durable succès, c'est sans doute parce qu'ils présentent des niveaux d'apprentissage allant du facile au plus difficile et s'adressent donc à un public ciblé ${ }^{14}$.

Cette attention accordée aux besoins des apprenants va de pair avec une interaction entre maitre et élève. Piélat, par exemple, pose comme objectif de faire réfléchir ses élèves sur des exemples et de trouver eux-mêmes les règles de grammaire :

Je ne fai que recreër les esprits, en ne les occupant que sur des exemples, à l'occasion desquels ils peuvent sans peine former eux-mêmes des préceptes, si tant est qu'ils ayent la curiosité de faire la fonction des Grammairiens (Sommaire).

Le maître doit être ouvert aux élèves, accepter voire stimuler leurs questions : ce principe est énoncé explicitement par du Casquet dans son Discours préliminaire ${ }^{15}$ : 
C'est pour vous instruire, en vous amusant que j'ai composé ce petit traité, que j'ai dessein d'augmenter par d'autres petits volumes, si je trouve qu'il soit gouté, et qu'il produise l'utilité que j'en espère. Je lui donne le titre d'entretiens par ce que j'y fais parler les écoliers et le maitre. Il faut que l'écolier ait la liberté de s'informer au maitre, de ce qu'il ignore encore, et il n'y a qu'un maitre ignorant dans sa suffisance, qui se puisse formaliser des questions curieuses d'un écolier avide à s'instruire.

La réputation du maître est fondée sur la qualité de son enseignement et elle est confirmée par ses publications qui peuvent d'ailleurs toucher à d'autres domaines que celui de l'enseignement de la langue: c'est ainsi que Parival publie plusieurs descriptions géographiques ${ }^{16}$ et un ouvrage d'histoire contemporaine, Piélat une vie de Michiel de Ruyter et Baudet, divers ouvrages de mathématiques ainsi que des brochures historiques ${ }^{17}$. Les manuels, les méthodes contribuent naturellement à la renommée du maître au-delà des frontières géographiques et temporelles. Les grammaires et dialogues de Mauger connaissent une double carrière anglaise et néerlandaise. Les dialogues de Parival ont connu une version franco-allemande ainsi qu'une adaptation italo-allemande. Quant à Marin, sa Méthode familière (1698) sera rééditée jusqu'en 1874, revue et corrigée bien sûr par les maîtres d'école du XIXe siècle qui, eux aussi, publient des méthodes à succès ${ }^{18}$.

\section{2b. La méthode du maître}

L'efficacité de la méthode repose aussi sur la simplification des règles de grammaire, la pratique de la conversation avec divers partenaires et des exercices variés, l'adaptation aux compétences et aux besoins de l'apprenant jouant là aussi un rôle essentiel.

21 Apprendre par la seule conversation et sans grammaire, voilà un plaidoyer pro domo de l'Abbé de Bellegarde pour qui «... nous voyons tous les jours des personnes qui apprennent les langues les plus difficiles sans grammaire, sans dictionnaire et sans aucune règle, par la seule conversation $»^{19}$, ou encore un pari lancé par le libraire éditeur de l'ouvrage de Piélat au titre quelque peu trompeur :

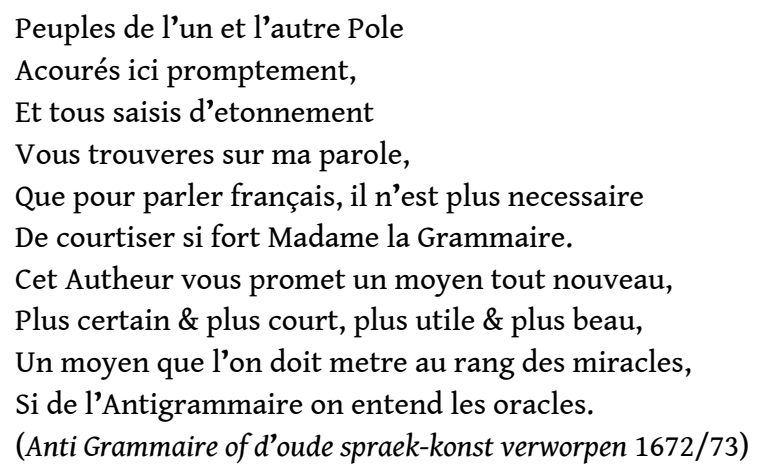

Ce qui pose problème c'est l'utilisation de la grammaire pour la grammaire et le mode d'apprentissage des règles. Piélat rejette la grammaire ancienne, proposant

[...]un Antidote contre le mal dont le monde se trouve atteint, en se servant des Grammaires, je veux dire contre l'Ennuy, le Depit, l'Impatience et le peu de profit qu'on en reçoit [... faisant] remarquer les abus des grammairiens, avec leurs preceptes confus et incertains (Sommaire).

Mais l'Anti-Grammaire ne signifie aucunement une absence de grammaire, car précise-til par ailleurs « je sçay bien qu'on ne peut pas s'engager à l'étude d'une langue, sans en cognoitre au préalable les élémens, de sorte que je n'ay pas eu dessein de les abolir ». 
En effet, si l'on reconnaît qu'une langue est faite pour être parlée, la connaissance des règles grammaticales est présentée comme nécessaire par ceux qui offrent méthodes ou dictionnaires de langue (Agron, 1835). Mais pour être efficace, la grammaire doit être simplifiée, comme l'affirment Piélat et Mauger qui fait le choix d'une présentation complète mais succincte des parties de la langue, donnant «les instructions nécessaires » d'une manière facile, car dans les « ouvrages pour servir d'introduction à la langue françoise [...] les règles [y sont] expliquées avec tant d'exceptions \& dans des termes si obscurs, qu'il est très difficile aus étrangers d'y faire quelque progrès qu'avec beaucoup de temps » (Avis, éd. 1700).

Pourtant le souci de simplification, à en croire $\mathrm{Chevalier}^{20}$, conduit Piélat à "s'embourber ", car malgré ses explications sur les verbes par exemple ${ }^{21}$, les règles de Piélat « ne se séparent guère de l'exposé traditionnel ». Sa méthode est selon Chevalier fondée sur "l'usage» que traduisent dialogues, proverbes et entretiens, une "Grammaire idéale, qui se dégage tout naturellement des exemples »"2. C'est en effet l'usage qui est pour lui le paramètre décisif pour les choix qu'il fait :

[parlant de la] Reigle principale, à sçavoir que du sert pour la chose définie, et de pour l'indéfinie, [il affirme qu'] il y a tant d'Exemples contraires à ceste Reigle, qu'il faut enfin l'oublier aussi bien que les autres pour ne s'atacher plus qu'à l'Usage : et afin que cet Usage ne soit pas trop long, il faut que vous appreniés des Exemples sur les plus diverses applications de ce du et ce de. C'est là l'unique moyen de vous en servir bientost et avec pureté et facilité (9).

Il faut présenter les règles de façon accessible, proposer une façon simple de les appliquer mais il faut aussi tenir compte du public apprenant et de ses besoins. Par exemple le souci d'enseigner la langue française de façon appropriée à un public qui ne connaît pas le latin est déjà présent au XVII ${ }^{e}$ siècle : "Je ne conseilleray jamais à un marchand qui n'a point de latin, ni à des filles et des femmes de se servir de la voye des grammaires : parce que les termes les épouvantent et qu'ils ne sçauraient les retenir sans une peine indicible, par faute d'en cognoitre leur origine latine ou grecque", explique Piélat parlant de sa méthode dans le discours enchassé au Dialogue XXXV (160). L'Anti-Grammaire de Piélat s'adresse en effet explicitement «aux Personnes de Negoce, qui sçavent quel est le profit, l'honneur \& le plaisir de parler les Langues étrangères » (Adresse du libraire, 1673).

L'activité de l'élève est là aussi, gage de toute efficacité. A lui de se fabriquer sa propre grammaire, comme le dit un élève chez Piélat : "Mon maistre m'en a fait faire une à moy mesme pour mon usage » (dialogue XIII, 61) ou comme le conseille Charrière à son neveu néerlandais Willem-René : "... vous vous en serez fait une [grammaire] dans votre tête que vous appliquerez à tout ce qui se présentera (O.C. V, 593) ». Cette attitude que l'on rencontre donc très tôt se développe au long du XIXe siècle; ainsi, pour Baudet ${ }^{23}$ « les règles sont des résultats de l'expérience et de l'observation. Je n'ai donc pas prétendu établir des règles préalables sur la formation des temps, et enseigner ensuite les conjugaisons par la synthèse " (Exercices II, [1834] $7^{\mathrm{e}}$ éd. 1845, 7). Cette méthode active, illustrée par Baudet dans ses livres d'exercices (1834-1836), inscrit l'apprentissage de la grammaire dans des exercices variés, de conjugaison, d'analyse, d'acquisition du vocabulaire. Si l'élève semble comprendre mieux qu'il ne parle, comme on le lui fait dire dans de nombreux dialogues ${ }^{24}$, signe de lacunes de l'enseignement de la grammaire, on doit pourtant remarquer que le débat sur la grammaire a opposé grammaire théorique et grammaire appliquée, induite par la pratique et par la conscience de la règle obtenue par l'observation de l'apprenant. 

da pratique de la conversation qui peut se faire en Hollande même où nombreuses et diverses sont les personnes de langue maternelle française, ou en France. Les modèles de conversation rassemblés par Bellegarde offrent des recettes pour des situations de rencontres puisque « le commerce des honnêtes gens est une bonne école, et que leur entretien peut tenir lieu d'étude ... le monde est un grand livre» (272-274). Selon Mauger, l'on peut apprendre le français « en France, à Paris ou à Blois ou bien à La Haye, où il y a tant de Français" (Dialogue XXII, 238); dans ses modèles de conversation galante, il encourage à "parler hardiment avec tout le monde et sur toutes sortes de sujets, en évitant de parler toujours [sa] langue maternelle », ce qui semble être la clé de la réussite, comme l'affirme le maître (Cazelles, 1809, 140). S'il "faut que la prononciation soit enseignée par un maître », on peut aussi s'exercer avec des locuteurs natifs :

Comment faites-vous pour apprendre la bonne prononciation?

- Je fréquente des Français qui prononcent bien,

- C'est ce que vous pouvez faire de mieux ...

- Parlez-vous souvent français?

- Quelquefois, quoique je le parle encore bien imparfaitement,

- Pour apprendre à bien parler, il ne faut pas craindre de parler mal ... (Peel, 1861, 81-82). la Loire, célèbre pour la pureté de la langue. Enfin il est très utile de varier les partenaires de la conversation.

En fréquentant des gens savants on acquiert toutes sortes de connaissances, le cadre culturel garantissant les normes linguistiques (Parival, 96), mais la fréquentation de gens simples est tout aussi utile, car converser en français en divers milieux permet de se familiariser avec divers registres de langues, d'acquérir un vocabulaire varié et spécifique et une bonne prononciation, et des compétences socio-culturelles et de communication. Ainsi, Mauger (Dialogue XXII, 238) recommande de «pratiquer les hommes de cour, qui viennent voyager en Hollande, aller souvent chez les Ambassadeurs de France et entendre les sermons ", Piélat trouve bénéfique d'avoir une gouvernante française (Piélat, 26) et Charrière un perruquier auprès de qui apprendre le bon accent (O.C. II, 458). Les dames sont elles aussi des partenaires utiles mais à manier avec précaution :

Documents pour l'histoire du français langue étrangère ou seconde, 33/34 | 2005 
[la fréquentation] des dames est aussi fort profitable, parce que ce sexe ne demeure pas longtemps muet. - Il est bien vray, mais il fait perdre beaucoup de temps, \& puis il engage à l'amour - C'est de quoy il faut se garder, car nous ne voyageons pas pour faire l'amour, mais pour apprendre la manière de vivre (Parival, dialogue $21 \mathrm{Du}$ voyage en France 105-106).

32 La qualité des partenaires de la conversation est garantie par le niveau de civilité des locuteurs, que leur langue soit l'apanage de leur milieu social et professionnel (diplomates, prédicateurs) ou de leur formation intellectuelle (savants); elle peut l'être aussi par le contexte dans lequel s'inscrit l'échange encouragé par la simplicité ou la familiarité. Ainsi, si l'on peut pratiquer la conversation en français dans son pays, il est tout à fait souhaitable de la pratiquer aussi en situation d'immersion sinon de contact linguistique et culturel en France, ce que permet le voyage, dans la tradition du Grand Tour.

33 Aux règles de grammaire et à la pratique de la conversation s'ajoutent les exercices basés sur la mémorisation, l'application des règles et la traduction. "Je crois que la seule manière d'apprendre vite une langue, c'est de mettre en pratique dans la conversation, tous les mots, les dialogues, et les phrases que l'on a appris par cœur ", affirme l'élève (Cazelles, 1809, 140). Mais les pratiques habituelles sont fort critiquées, car bien loin de l'idéal: "c'est bien malheureux que la plupart des maîtres ne comprennent pas que quand on saurait des volumes entiers de dialogues et de phrases, on n'en serait guère plus avancé, lorsqu'il s'agiroit de discourir en société ou d'écrire " (Cazelles, 1809, 154). Ces considérations illustrent bien ce qui peut apparaître comme contradictoire dans la pratique du « par cœur».

34 Apprendre par cœur est réputé fatigant (Peel, 1861, 81) et même fastidieux, mais il est considéré comme indispensable (Agron, préface au Dictionnaire portatif, $3^{\mathrm{e}}$ éd., 1835), d'apprendre par cœur des règles, des mots, des textes entiers (L'Art poétique de Boileau, pour Isabelle de Charrière), car "il est aussi impossible d'apprendre une langue étrangère quelconque sans faire provision de mots, qu'il est impossible d'élever un édifice sans rassembler les matériaux qui doivent servir à la construction " (Phrases faciles, 1804, Avertissement ${ }^{26}$ ). Pour rendre efficace la mémorisation, il convient de varier le contexte dans lequel reviennent les mots et expressions, et d'alterner les exercices. Baudet pour qui l'élève doit être appelé «à comparer et à généraliser », à partir de «ce qu'il a appris par cœur ", dit à ce sujet

[avoir adopté] la méthode du charpentier et [s']en tenir à l'équarrissage, [ayant] compris qu'au dix-neuvième siècle, il ne faut pas enseigner une langue vivante, comme on enseignait le Latin et le Grec au seizième, en faisant apprendre toutes les règles et toutes les exceptions relatives au substantif avant d'oser toucher à l'adjectif. [... Il offre donc à l'élève] une série d'occupations propres à exercer en même temps son jugement et sa mémoire; tandis que la simple imitation et même un peu de routine trouvent leur part dans son travail (Exercices IV, [1834] 1837, Avant-propos).

La clé de l'efficacité de l'apprentissage par la mémorisation réside sans doute dans la variation et la répétition des exercices pour appliquer les règles apprises. Fournier (Préface, $1810,1^{\mathrm{e}}$ éd. 1801, 83) conseille " aux maitres de faire expliquer tous les jours à leurs élèves dix à douze lignes de parties du discours, avant de terminer leurs leçons ». Et la mise en scène d'un élève aux prises avec les exercices d'apprentissage du français (Peel 1861, 81-82) montre une démarche complexe et très sérieuse, avec apprentissage par cœur des règles, lectures d'oeuvres littéraires et exploitation très consciencieuse 
des possibilités d'investigation, d'appropriation de la langue par recherche de sens, explication et traduction.

Enfin la traduction apparaît comme un élément essentiel dans la chaîne de l'apprentissage. D'un côté, le savoir traduire semble être la compétence ultime pour la connaissance de la langue étrangère ; de l'autre, l'exercice de traduction est ce qui mène à cette connaissance. Aussi l'exercice de traduction est-il partout présent, et sinon, il est préférable de changer d'école si l'on y fait jamais de thèmes car «ce n'est qu'à force d'expliquer et de traduire, tant d'une façon que de l'autre, qu'on apprend une langue » (Cazelles 1809, 154-155). Charrière rappelle à son neveu Willem-René que son père obligeait ses frères à traduire quotidiennement du français en hollandais et du hollandais en français et elle lui donne des exercices de traduction à faire: Cornelis Nepos à traduire du latin en français, et Wagenaar à traduire du néerlandais en français.

$\mathrm{Au}$ centre de l'exercice de traduction se trouvent la problématique de l'idiome et l'acquisition d'une compétence plus culturelle que linguistique. Agron (Préface à la $1^{\mathrm{e}}$ éd., $3^{\mathrm{e}}$ éd. 1835) propose aux élèves de traduire un texte français en néerlandais puis de traduire ce texte néerlandais en français, s'efforçant de retrouver le texte original. Les idiomes (gallicismes, néerlandicismes) mis en évidence pourront ainsi être expliqués et plus facilement mémorisés grâce au Dictionnaire... qui remplacera auprès des élèves les «dialogues sans sel». Pour Baudet, traduire est un exercice complexe dans lequel l'apprenant doit apprendre à distinguer la norme et l'usage, la forme et le fond ; aussi combine-t-il les exercices en accrochant aux textes de thèmes et versions donnés à traduire, des exercices d'application grammaticale, de conjugaison, d'analyse, enfin d'acquisition du vocabulaire.

La pratique du maître de langue est une didactique de terrain, au centre de laquelle se trouve l'élève avec toutes ses capacités à développer grâce aux exercices. La modélisation de cette pratique et une théorisation élémentaire de la discipline lisible en filigrane dans les préfaces se font sur la base de l'expérience et sont mises au service d'une formation éducative.

\section{2c. La formation plurielle dispensée par le maître de langues}

L'apprentissage de la langue est un travail disciplinaire par ses objectifs et ses méthodes : l'apprenant acquiert des compétences (savoir parler) au moyen d'exercices, mais le maître reste longtemps un maître dans plusieurs disciplines ou plusieurs langues et le français peut servir de substrat à la formation scolaire et éducative et à l'enseignement de matières spécifiques.

Le maître enseigne certes au premier chef la langue française, mais il est assez courant qu'il enseigne aussi celle du pays où il s'est expatrié : Mauger a commencé à enseigner l'anglais à Londres à de nobles étrangers de passage et, de retour à Paris vers 1680, il est probablement maître d'anglais. Marin, qui arrive à Amsterdam en 1688, y est actif pendant trente ans comme maître de français et de néerlandais. En outre leurs ouvrages sont censés permettre l'apprentissage et de la langue étrangère et de la langue maternelle, comme l'indiquent souvent les pages de titre (Marin, Nouvelle méthode, 1694) ou l'Avertissement au lecteur (Phrases faciles..., 1804).

41 Le plurilinguisme est également assez fréquent. S'il faut peut-être faire la part de l'exagération chez Piélat qui, d'après la page de titre de la seconde édition de son AntiGrammaire (1681), serait professeur de langues hébraïque, grecque, latine, italienne, 
française, allemande, hollandaise et anglaise, de même que de rhétorique, de philosophie, de théologie et de médecine, on trouve surtout à partir de la fin du XVIII siècle un certain nombre de maîtres qui pratiquent souvent le plurilinguisme: ainsi Fournier est professeur de langue française, latine, anglaise et allemande. Engelberts Gerrits (1795-1881) est maître d'école française avec internat à Amsterdam et il y enseigne également l'anglais. Gilbert, qui adapte dès 1775 la Nouvelle méthode de Marin, est maître de langue latine, française, anglaise et néerlandaise ${ }^{27}$. Quant à du Casquet, maître dans une école française à Dordrecht de 1791 à $1794^{28}$, il dispense un enseignement plurilingue (anglais, allemand, italien) mais accorde une place privilégiée au français qui occupe non seulement la place de première langue étrangère, mais joue aussi le rôle de langue d'enseignement, comme on peut le lire dans l'Avant-propos de ses Entretiens (1792) :

La langue française étant, de celles que j'enseigne dans mon école pensionnaire, la première, la plus generale et par laquelle j'entreprens tous mes écoliers; j'ai crû aussi devoir écrire en cette langue-ci. J'enseigne aussi, l'Anglais, l'Allemand et l'Italien, avec des sciences necessaires pour cultiver l'esprit de la jeunesse. Mais je ne passe jamais à d'autres choses, qu'avec des écoliers qui sachent déjà le Français au point, qu'en leur enseignant autre chose, la langue française puisse servir pour leur expliquer le reste.

Le plurilinguisme de ces maîtres s'explique par les objectifs mêmes de ces écoles françaises qui forment les enfants de la bourgeoisie se destinant au commerce et pour qui les langues vivantes et l'arithmétique sont particulièrement importantes; il correspond également à l'évolution que connaît l'enseignement à partir de la fin de XVIII ${ }^{e}$ siècle, un enseignement qui doit s'ouvrir au monde moderne, en particulier grâce à l'étude des sciences et des langues vivantes étrangères.

Par ailleurs, en enseignant la langue, le maître communique d'autres savoirs en particulier la géographie et l'histoire; en témoignent les Dialogues de Parival, les Dialogues françois \& flamends de Mauger ou la Nouvelle grammaire augmentée de Nouveaux Dialogues \& de lettres nouvelles de Marin qui sera imité à la fin du XVIIIe siècle par Cazelles. On trouve également, mais de façon moins systématique que pour la géographie et l'histoire, des dialogues et des exercices où sont dispensés des savoirs scientifiques, sous forme de notions de physique, de sciences naturelles, d'astronomie ou bien des savoirs professionnels concernant la navigation et le commerce. Ainsi, Mauger, dans la seconde partie de ses Dialogues françois et flamends (1700), fait converser deux gentilhommes sur le soleil (XX, 232 et sq.) ou bien encore deux hommes de lettres sur " la nature des plantes " (XVIII, 225 et sq.). Piélat, dont l'ouvrage s'adresse " particulièrement aux personnes de négoce», insère un dialogue " Au sujet des lettres de change \& de la bourse» (XXVIII). Les ouvrages de Marin comportent aussi des dialogues traitant du commerce, de la bourse, des lettres de change, etc. Les dictées et les vocabulaires peuvent aussi fournir l'occasion de dispenser ces savoirs : dans les Dialogues français, hollandais et anglais sur des sujets familiers à l'usage des écoles (1803), les dictées françaises que doit faire l'élève ( $26^{\mathrm{e}}$ dialogue,133-139) portent " sur la géographie, l'astronomie, la physique, ou le commerce ", tandis que dans la préface du Recueil de mots hollandais-français $(1851)^{29}$, l'auteur affirme que "pour donner à cet ouvrage toute l'utilité dont il était susceptible, [il a] consacré au commerce, à la navigation, à l'industrie, aux arts et aux sciences, etc. des articles intéressants ». Ces savoirs restent bien entendu relativement succincts, mais on ne manque pas de préciser qu'il est indispensable de compléter, aussi bien pour améliorer la pratique de 
la langue que pour développer ses connaissances, cette première formation, en particulier par les lectures, comme nous le verrons plus loin.

Par le biais de l'apprentissage de la langue, le maître initie aussi à l'usage du monde. Lorsque l'on lit les dialogues de Parival rédigés à l'origine pour de jeunes nobles allemands qui faisaient leurs études à l'université de Leyde, ou ceux de Mauger, on voit se dessiner en filigrane le modèle d'éducation aristocratique dont le Grand Tour fait partie intégrante. Le perfectionnement de la langue française qui constituait l'un des objectifs majeurs de ce modèle éducatif ${ }^{30}$, s'inscrit dans un ensemble de pratiques, danse, armes, équitation, musique, toutes nécessaires pour acquérir l'aisance requise dans la bonne société. Inculquer l'art des bonnes manières et de la conversation, dans lequel les Français ont la réputation de briller, est donc aussi l'un des objectifs de ces dialogues et aussi celui de l'abbé de Bellegarde qui dans ses Réflexions sur ce qui peut plaire ou déplaire dans le commerce du monde (1709) propose des modèles de conversation pour " les personnes polies ». De ce modèle aristocratique, se dégage le profil d'un maitre dispensateur de culture mondaine, maître dans l'art de converser, comme l'a montré aussi Nadia Minerva à propos des manuels de français utilisés en Italie aux XVII ${ }^{e}$ et XVIII ${ }^{e}$ siècles ${ }^{31}$. Ces références aristocratiques ne se trouvent ni chez Piélat $^{32}$ ni chez Marin. Cela peut tenir au milieu social des apprenants auxquels s'adressent ces deux auteurs, mais aussi à l'âge des utilisateurs : les conversations galantes proposées par Mauger ne sont sans doute guère appropriées à de jeunes élèves. Aussi dans les manuels utilisés dans les écoles, la formation mondaine passe-telle au second plan et l'apprentissage de la langue est lié à celui des valeurs morales et religieuses. Là réside pour du Casquet (1792) la vocation du maître d'école qui « est d'instruire et de former l'esprit de la jeunesse, en plusieurs sciences, et son cœur en l'amour de la religion et de la vertu » (Avant-propos). Et ses entretiens qui mettent en scène maître et écolier traitent, à l'exception du dernier consacré au négoce, essentiellement de sujets religieux et moraux. Les méthodes de Baudet très populaires tout au long du XIXe siècle, associent également l'apprentissage de la langue à celui de la morale et de la vertu ${ }^{33}$. Le maître contribue donc à la formation de l'apprenant sur le plan cognitif, social et moral, et ce faisant, participe à la construction identitaire de ce dernier.

\section{Le maître de langues médiateur social, éducatif et culturel}

Le maitre tente de joindre l'utile à l'agréable pour le profit des élèves. Il leur fait découvrir la langue et la culture étrangères mais aussi leur propre monde. En ce sens il est un acteur de la formation identitaire de ses apprenants.

\section{3a. Utile dulci, règle d'or du bon maître}

Utile dulci, joindre l'utile à l'agréable, ce principe emprunté à l'Art poétique d'Horace ${ }^{34}$ qui règne en maître dans les lettres et les arts de la Renaissance au Romantisme est aussi celui de nos maîtres de langue. Il y est fait explicitement référence dans l'Avis au lecteur des Dialogues françois \& flamends de Mauger par le libraire qui affirme :

Si jamais personne a mérité qu'on dise de luy, au sujet de la Langue Françoise, qu'il

surpasse tous les autres, \& qu'il mêle l'utilité avec le plaisir, selon le verset latin, 
Omne tulit punctum, qui miscuit utile dulci

C'est asseurement Claude Mauger. contemporain, qui se manifeste par des allusions ponctuelles à des personnages connus voire célèbres ${ }^{36}$ ou par les références à des faits d'actualité, qui ne se bornent pas d'ailleurs au cadre strictement français. Mauger consacre un dialogue entier à la guerre avec les Turcs et au siège de Candie ${ }^{37}$ (dialogue 27), un autre à la peste qui sévit à Paris (dialogue 45) tandis qu'au XIXe siècle, Baudet évoque, dans des textes de thèmes 
(Exercices VI, [1836] $7^{\mathrm{e}}$ éd. 1873,122-124) la comète de Halley « vue au mois d'octobre 1835 ». L'intérêt porté au contemporain est aussi manifeste dans les faits-divers rapportés sous le titre d' " histoires $»^{38}$ qui constituent des lectures complémentaires aux dialogues, comme le propose Parival : «Après les avoir appris [les Dialogues], tu pourras lire les Histoires suivantes [Histoires tragiques de nostre temps, arrivées en Hollande] avec quelque emolument \& plaisir » (1656, Avertissement au lecteur).

Ces connaissances encore assez élémentaires sur la culture étrangère sont à approfondir par des lectures. Quels livres recommandent le maître? Des livres d'histoire, des livres de philosophie et de beaux romans. Mauger précise quelques titres :

Quel autheur lisez-vous?

Nous lisons Pharamond

Et moy, je lis l'histoire de Francion. C'est une histoire fort plaisante.

Avez-vous le Grand Cyrus? (dialogue 26, 63)

53 Ce sont les romans à la mode, lectures qui, comme celle du Grand Cyrus ${ }^{39}$, initient à la littérature précieuse et à la langue raffinée de la bonne société, tandis que le roman burlesque de Ch. Sorel, Histoire comique de Francion ${ }^{40}$ donne accès au contraire à toute une diversité de registres de langue. Aux XVIII ${ }^{e}$ et XIXe siècles, les romans sont moins souvent recommandés : considérés comme une lecture frivole, voire corruptrice ${ }^{41}$, ils suscitent la méfiance des éducateurs et on leur préfère les ouvrages d'histoire et surtout les grands auteurs du classicisme français: Molière, Racine, Boileau, La Fontaine, Fénelon. C'est ainsi que Charrière s'agace du goût trop prononcé de son neveu pour les romans et lui recommande de lire Les Aventures de Télémaque de Fénelon pour améliorer son style (O.C., IV, 509, V. 314-315). Au XIX e siècle, les maitres d'école reprennent ces grands noms du répertoire classique et y ajoutent ceux du Siècle des Lumières, Voltaire, Buffon, Raynal, Marmontel, Bernardin de St Pierre, dans les exemples utilisés pour expliquer les règles ou dans les textes proposés comme exercices de traduction.

On constate aussi une autre évolution : Au XVII ${ }^{e}$ siècle, la découverte de la culture étrangère par le biais des lectures peut aller jusqu'à une tentative d'assimilation, comme le suggère le libraire aux futurs utilisateurs de l'Antigrammaire de Piélat qui, après avoir, grâce à la méthode :

[...]acquis toute la Langue Françoise , [pourront] faire une bonne provision de livres François les plus rares; \& par ce moyen metamorphoser [leurs] esprits en des esprits François, autant qu'il en sera de besoin pour [leur] profit, [leur] honneur, \& [leur] plaisir.

Aux XVIII ${ }^{e}$ et XIX ${ }^{e}$ siècles, les maîtres visent par la lecture des bons auteurs à améliorer le niveau de français de leurs élèves, à leur faire acquérir des connaissances, à les initier également à la morale comme l'annonce clairement le titre d'un ouvrage tel que Leçons françaises de littérature et de morale, ou choix de morceaux extraits des meilleurs auteurs français (1836-1837). Mais il ne s'agit plus de métamorphoser leurs esprits en esprits français ni de leur proposer le modèle de civilité à la française, mais d'en faire de bons Néerlandais, comme Isabelle de Charrière l'attend de son neveu : elle lui déconseille fortement de s'efforcer à acquérir l'art - français - de converser spirituellement sur des riens, incompatible selon elle avec le caractère néerlandais dont les vertus sont la simplicité, le naturel, la solidité et le sérieux ${ }^{42}$. Ce sont là les vertus que les maîtres d'école française s'efforcent d'inculquer à leurs élèves en même temps que la connaissance de la langue française, mais à la différence de Charrière qui 
s'intéresse à quelques individus, il s'agit pour eux de former aussi des citoyens par une éducation qui doit toucher le plus grand nombre. L'apprentissage de la langue étrangère implique donc aussi la construction identitaire par rapport à soi.

\section{3c. La construction d'une identité propre via la langue de l'autre} de traduction est formateur pour une meilleure connaissance de la langue maternelle ${ }^{43}$. La méthode de Marin est conçue «pour apprendre les principes \& l'usage des langues françoise et holllandoise ( $8^{\mathrm{e}}$ éd., 1812). Ceci est bien entendu excessif puisque c'est la langue française qui, centrale, est l'objet d'étude, mais on voit poindre dès le XVII siècle ce souci de comparatisme linguistique et culturel, d'apprentissage raisonné. Ainsi le dictionnaire de Marin comprend-il «tous les mots de l'usage avouez de l'Académie française \& autres auteurs d'élite, exactement définis et clairement expliquez par des

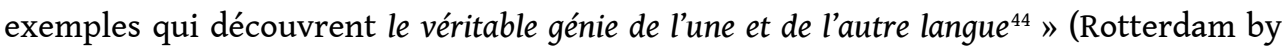
Hendrik Beman, 1758).

Pour les auteurs de méthodes qui participent à la constitution d'une discipline, l'apprentissage de la langue étrangère inscrit l'apprenant dans une démarche de raisonnement, d'analyse, de comparaison; ceci a des incidences sur la connaissance (scientifique) de la langue maternelle et sur la compréhension de ce qu'est une langue et son fonctionnement en contexte. Ce processus se poursuit au XIX ${ }^{e}$ siècle, les positions de l'auteur étant lisibles dans les textes donnés en exercices:

Chaque langue a ses misères et ses ressources; chaque peuple a sa manière de voir et de s'exprimer. Il est donc souvent impossible de traduire une phrase mot-à-mot, soit qu'un mot d'une langue n'ait point d'équivalent dans une autre, soit qu'un peuple repousse comme inusitées des expressions approuvées par ses voisins (Baudet, Exercices VII, 1842, 5).

Baudet s'efforce par ailleurs tout au long de ses Exercices de faire saisir à l'élève les différences par rapport à son champ linguistique et culturel, sur le plan des structures grammaticales, des usages, des références, ponctuant avec "pour un Hollandais ... comme en hollandais ». Ceci participe de tout un effort pour favoriser la construction identitaire par une démarche contrastive et un apport de connaissances en miroir, sur soi en passant par l'autre.

Les dialogues et autres textes d'exercices donnés pour la traduction apportent à l'apprenant des connaissances sur sa culture maternelle. Les textes de manuels regorgent d'informations de toutes sortes qui apportent à l'élève un savoir de base, des connaissances élémentaires sur la géographie et l'histoire de son pays, sur les activités 
que l'on y rencontre. La géographie est abordée au fil de récits de voyage ${ }^{45}$, l'histoire des Provinces-Unies fait l'objet de dialogues ${ }^{46}$ dans les méthodes familières ou les livres d'exercices; on la trouve aussi sous la forme de tableaux chronologiques ${ }^{47}$ publiés par les maîtres des écoles françaises.

Nous retrouvons donc les mêmes éléments que nous avons relevés en ce qui concerne la connaissance de l'autre, mais cette fois ayant trait aux Pays-Bas; Parival, avec les Histoires tragiques de nostres temps, arrivées en Hollande ... (Parival 1656) ancre son enseignement dans un contexte contemporain et local. Mais au XIX ${ }^{\mathrm{e}}$ siècle, cette mise en scène des Hollandais et de la Hollande véhicule des images plutôt positives (les villages de Frise sont riants) et des valeurs morales (l'amour de la patrie est une qualité de la nation hollandaise, Baudet, Exercices VI [1836] $7^{\mathrm{e}}$ éd. 1873, 3, 20). Un texte de Raynal donné comme exercice de version est particulièrement éloquent :

La république de Hollande offre en naissant un grand spectacle aux nations, et doit rester un puissant objet d'intérêt pour nous, et de curiosité pour notre postérité la plus reculée ... Les Hollandais ne faisaient pas toujours de grandes choses ; mais ils n'en faisaient pas d'inutiles (ib. 107-108).

On mesure ici la différence avec le XVII ${ }^{\mathrm{e}}$ siècle. On assiste en effet au XVIII ${ }^{\mathrm{e}}$ siècle à une prise de conscience de l'héritage national et à l'exaltation du patriotisme hollandais en opposition à la francisation poussée des élites. La place du pays dans l'histoire et la grandeur du siècle d'Or sont réévaluées. On revendique alors une identité nationale qui s'appuie sur des faits et des hommes du patrimoine historique, alors qu'au XVII les grands hommes et les faits que l'on vante sont ceux du moment, de la cour (Mauger). Marin prend la suite de Mauger et remplace le dialogue consacré à l'histoire de France par un dialogue consacré à l'histoire des Provinces-Unies. Et plus tard Baudet intègre de façon très naturelle dans ses exercices, les savoirs - géographie, histoire, pensée, sciences - sur le pays de l'apprenant. Un texte de version, signé Condillac par exemple, pointe des hommes, des lieux et des activités :

Rudolphe Agricole, d'un village près de Groningen, avait commencé à répandre la littérature ancienne en Allemagne, lorsqu'Erasme, né à Rotterdam vers l'an 1647, faisant ses études à Deventer, sous Hegius, disciple d'Agricola (Exercices III, [1834], $14^{\mathrm{e}}$ éd. 1857, 198).

63 Dans la langue de l'autre sont exprimés des éléments qui appartiennent à l'un et à l'autre. Les principes moraux véhiculés par les dialogues, textes d'exercices ou exemples de grammaire expriment dans la langue de l'autre les contours d'un patrimoine qui ainsi devient commun. Les citations pléthoriques de La Fontaine, Boileau ou Fénelon dessinent pour l'élève un comportement modèle, une morale à suivre. C'est en langue française que sont exposés ces comportements idéaux donnés en image aux élèves hollandais, par le biais de citations de ces auteurs classiques ou dans des textes composés pour les besoins de la cause. Ainsi, des textes de versions se prêtent facilement à ce propos :

Les Demoiselles estimables trouvent dans leurs occupations journalières les moyens d'être utiles et de captiver le respect de leurs connaissances [...] ; elles n'étudient ni les langues anciennes, ni la jurisprudence. Elles se contentent de se perfectionner dans les langues modernes. Elles s'exercent dans la géographie, l'histoire, le dessin, la musique ; n'oublions pas la danse. D'un autre côté, elles tricotent des bas, brodent des bonnets, soignent le linge, aident leur mère dans les travaux du ménage, s'appliquent à connaître les ouvrages de la cuisine, et se persuadent qu'en s'accoutumant à toutes ces choses, elles sauront un jour bien diriger un ménage (Exercices II, [1834], $7^{\mathrm{e}}$ éd. 1845, 97-98). 

responsable, comme on peut le lire dans un texte d'exercice :

Nous naissons capables d'apprendre; mais ne sachant rien, ne connaissant rien. Comment donc apprenons-nous ? Les leçons d'un maître peuvent nous instruire de ce que nous devons faire pour devenir habiles; mais il est nécessaire que nous parcourions nous-mêmes le chemin qu'il nous indique. La lecture faite avec réflexion, concourra avec les leçons du maître à développer nos facultés. Celui qui sait recueillir les idées répandues dans des livres choisis, s'instruit non seulement de ce que les grands hommes ont pensé, mais de ce qu'ils ont jugé digne d'être communiqué (Exercices II, [1834], $7^{\mathrm{e}}$ éd. 1845, 165-166).

Ainsi ce que l'on attend de l'élève se lit en transparence. On utilise l'autre pour construire en miroir une image de soi. Le texte proposé en thème et attribué à Raynal (voir ci-dessus) illustre bien ce mécanisme : l'image du Hollandais construite par un penseur français est offerte au jeune Hollandais pour développer en lui un processus de construction identitaire par rapport à l'image proposée.

Par ailleurs on trouve dans les textes de dialogues ou de versions, des passages concernant l'histoire de France et celle des Provinces-Unies, des aspects qui touchent à leur passé commun : un texte de Voltaire sur la mort de Coligny par exemple, des récits traitant de la présence (militaire) française aux Pays-Bas avec la guerre de Hollande menée par Louis XIV et la mémorable année 1672, et plus tard 1795 et les suites de la révolution (Baudet, Exercices VII ([1842], 4éd. 1860, 79-87).

Dans les manuels destinés à l'apprentissage de la langue française se trouvent donc un savoir linguistique et culturel (histoire, géographie, coutumes) sur le monde de l'autre, dans lequel l'apprenant est inclus par contraste ou par association (les normes sociale et morale étant par exemple similaires); celui-ci, par effet de miroir, lui est en fait proposé comme modèle pour soi. Mais le monde de l'apprenant y est lui aussi dessiné, géographie, histoire présente sous forme d'évènements et de personnages, réalités contemporaines: Cazelles (1854, $26^{\mathrm{e}}$ dialogue) mentionne l'explosion du vaisseau de poudre à Leiden en 1807 et l'assèchement du lac de Haarlem commencé en 1840 et achevé en 1853. L'apprentissage d'une mémoire commune élaborée à partir de l'histoire et de principes moraux partagés avec l'autre et abordés dans la langue de l'autre a pour objectif l'édification du futur citoyen.

68 Aux Pays-Bas, le professeur de langues n'a un statut institutionnel qu'à la fin du XIXe siècle : la discipline élaborée à l'université est essentiellement une étude historique et philologique, la formation professionnelle des enseignants du secondaire est absente jusqu'au début du XXe siècle. Pourtant, le maître de langues, tel que nous venons de l'évoquer, est un professionnel soucieux d'efficacité, conscient de son métier, réfléchissant sur sa pratique comme le montrent les méthodes qu'il rédige. Il enseigne une discipline, mais son action porte plus généralement sur l'éducation et l'instruction de ses élèves. Le maître de langues, passeur entre les deux langues, entre les deux cultures, contribue par ses manuels, par les exercices qu'il propose, à la construction identitaire de l'apprenant. 


\section{NOTES}

1. G.G. Baardman, « Geschiedenis van het onderwijs in vreemde talen tot het einde van de XIXe eeuw ", Levende Talen 172, 1953, 525-549.

2. En 1828 pourtant, le ministre de l'Intérieur de l'époque avait insisté sur l'importance d'une formation pédagogique pour les enseignants (Levende Talen 1911-1961, jubileumnummer Groningen, 1961).

3. M.-C. Kok Escalle, "Le français aux Pays-Bas dans la deuxième moitié du XIXe siècle ", Documents Sihfles, 23, 1999, 82-107.

4. M. Engelberts, «Bourgeoisie libérale et langues modernes: le débat parlementaire sur la création de chaires universitaires aux Pays-Bas, 1876-1885 », Documents Sihfles, 15, 1995, 38-51.

5. B. Sijmons, "Het onderwijs in de moderne talen», Academia Groningana 1914 Groningen Noordhoff, 416-437.

6. A.G. van Hamel, La chaire de français dans une université néerlandaise, Groningen J.B. Wolters 1884 ; J.J. Salverda de Grave, Quelques observations sur l'évolution de la philologie depuis 1884, Leide van der Hoeck , 1907.

7. B. Piélat, L'Antigrammaire du Sieur Barthélemy Piélat, ...adressée aux Messieurs et dames d'Amsterdam...D'Oude Spraek-Konst verworpen van den Heer Barthel Pielat...toe-geeigent aen de Heeren en Jufferen van Amsterdam...Amsterdam J. Jeansson de Waesberge, 1672/1673. Nous avons consulté l'édition de 1681.

8. P.J. Baudet, Fransche oefeningen met de noodige spraakkunstige aanwijzingen en ophelderingen, ten dienste der Nederlandsche Jeugd, Deventer J. de Lange, 1834-1836 (I-VI), 1842 (VII), 1844 (VIII) (Exercices).

9. Editions consultées : J.-N. Parival, Histoires tragiques de nostres temps, arrivées en Hollande et quelques dialogues françois, selon le langage du temps, Leyden N. Hercules, 1656. Dialogues françois, selon le langage du temps, Leyden N. Hercules, 1659. 2e éd. Dialogues françois, selon le langage du temps [. ..] Dixiéme et derniere edition; augmentée de l'Ecole pour rire, reveüe \& corrigée selon la méthode de Richelet, Leide Th. Haak, 1709.

10. Les réfugiés wallons qui dès le XVIe siècle s'installent comme maître de français dans les Pays-Bas septentrionaux, ont l'avantage de connaître le français et le flamand. Mais les Français de France ne sont pas toujours des modèles linguistiques pratiquant une langue conforme à la norme, étant donné les nombreux dialectes et patois.

11. Edition consultée: Grammaire françoise et flamende de Claude Mauger. Avec les dialogues du même auteur. Augmentez de nouvelles reflexions et modelles de lettres pour s'exercer tant en la traduction qu'en la composition, \&c.\&c. Tot Leyde by Frederik Haaring, 1700.

12. Il s'agit de La clavicule de la langue Françoise : par laquelle hommes \& enfans peuvent des couvrir \& aprendre facilement les fondements de la dite langue, \& aussi de la Flamende [...], Amsterdam Sander Wybrantsz, Harmen van den Berg, 1683.

13. P. Marin, Méthode familière pour ceux qui commencent à s'exercer dans la langue françoise... Gemeenzame leerwijze voor de geene die zig in 't Fransch beginnen te oefenen, Amsterdam héritiers de la veuve De Groot, 1698; Nouvelle méthode pour apprendre les principes et l'usage des langues françoise et hollandoise. Dat is, Nieuwe Fransche en Duitsche spraakwijze, Amsterdam Pieter Sceperus, 1694; Nouvelle grammaire françoise. Nieuwe Fransche grammatica ofte oprechte methode van Pieter Marin, Amsterdam, 1704 (P. Loonen, « Is die P. Marin onsterfelijk? Het succes van een vergeten taalmeester ", in Meesterwerk 8, 1997, 14-22).

14. P. Loonen, « Marin als maat voor de Franse les: een verkenning », in Meesterwerk 10, 1997, 23-28. 
15. J. Ebrard du Casquet, Entretiens du maître avec ses écoliers, Dordrecht aux dépens de Morks, 1792.

16. Les Délices de la Hollande (1e éd. 1651) entre autres servira de guide à des générations de voyageurs, tout au long des XVIIe- XVIIIe siècles et même encore au XIXe.

17. D.J. Beckers, M.-C. Kok-Escalle, Pierre Joseph Baudet (1778-1858), instituteur modèle de la première moitié du XIXe siècle, Nijmegen University of Nijmegen, Department of Mathematics, 1998 (Reports no 9816) p. 53-56.

18. La Nouvelle méthode familière pour l'usage de ceux qui veulent apprendre la langue françoise (1778) de Cazelles connait 21 éditions, les Principes de la grammaire française (1801) de Fournier, 16. 19. Modèles de conversations pour les personnes polies, rassemblés par l'Abbé de Bellegarde, Amsterdam : Schelte 5e éd., 1709, p. 272.

20. J.C. Chevalier, «De la grammaire générale au Traité de Regnier» in J. de Clercq, N. Lioce \& P. Swiggers (eds.), Grammaire et enseignement du français 1500-1700, Paris-Sterling (Virginia) Peeters, 2000, 575-576.

21. "J'ay fait un abrégé des verbes, tant réguliers que irréguliers : \& quand vous verrez l'ordre dans lequel j'ay mis les conjugaisons, vous avouërez que j'ay rendu tout à fait facile ce que l'on a toujours ouî le plus difficile de la langue françoise, attendu que dans huit petits articles j'ay renfermé toute la conjugaison, en y comprenant les deux mots de la fin, \& celuy du commencement, auquel tous les autres aboutissent, comme les membres à leur chef, ou les branches à leur racine. De plus, on n'aura pas beaucoup de peine à apprendre les verbes irréguliers, si l'on prend garde, que les irrégularités sont imprimées d'un autre caractère que le verbe régulier Porter » (Sommaire).

22. Si la 1e édition (1672/73) est essentiellement centrée sur l'oral par son offre de dialogues, la 2e (1681) offre, elle, des règles pour la prononciation et la grammaire (156 pages supplémentaires) complétées par du vocabulaireet des entretiens; en outre l'approche inductive qui caractérise la 1e édition est abandonnée dans la $2 \mathrm{e}$; cf. P. Loonen, "The influence of the Huguenots on the teaching of French » in J. de Clercq, N. Lioce \& P. Swiggers (eds.) 2000, op. cit. p. 323-325.

23. M.-C. Kok Escalle, «Les Exercices français de Pierre Joseph Baudet », Documents Sihfles 24, 1999, 161-173.

24. 26e dialogue des Dialogues français, hollandais et anglais sur des sujets familiers à l'usage des écoles, Rotterdam by W. Locke et cie, 1803.

25. C'est encore le cas aujourd'hui où l'Institut de Touraine, à Tours, est toujours très prisé pour l'enseignement du français aux étrangers.

26. Phrases faciles tres-necessaires pour faire traduire aux commençants, avant les mettre aux thèmes de la methode familière de P. Marin, nouvelle édition revue et corrigée, Leiden D. du Mortier et fils, 1804.

27. Nieuwe Fransche en Nederduytsche Spraakwijze, vermeerderd met een uitvoerige Syntaxis of Woorden-schikking. Den laatsten druk. Op nieuws overzien...door J.-J. Gilbert, leermeester in de Latijnsche, Fransche, Engelsche en Nederduitsche Taalen, Amsterdam H. Botter, 1775, 1790, 1800.

28. C. Esseboom, Onderwysinghe der jeught. Onderwijs en onderwijstoezicht in de 18e eeuw op het eiland van Dordrecht, Ridderkerk, 1995, p. 301.

29. D.W. Bosch, Dz., Recueil de mots hollandais-français rangés par ordre de matières. Premier livre d'étude pour les classes supérieures des maisons d'éducation et les gymnases, Amsterdam Schalekamp, van de Grampel \& Bakker, 1851.

30. A. Frank-van Westrienen, De Groote Tour. Tekening van de educatiereis der Nederlanders in de zeventiende eeuw, Amsterdam Noord-Hollandsche uitgeversmaatschappij bv, 1983 p. 152.

31. N. Minerva, « Le profil du maître à travers les dialogues et les lettres des manuels de français à l'usage des Italiens (XVIIe et XVIIIe siècles) », Documents Sihfles, 9, 1992, 5-9. 
32. Piélat (éd. 1681) insiste sur la dimension religieuse, faisant de fréquentes allusions à la lecture de la Bible dans les dialogues pour débutants : « Quel livre lisez-vous? Point d'autre que la Bible. Il faut lire l'Ecriture Sainte en français. Je porte toujours à l'Eglise un nouveau Testament françois » (dialogue 2, 25-26) ; "Où avez-vous mis ma Bible? Alles la querir \& lises un chapitre devant moy $»$ (dialogue 7, 42).

33. D.J. Beckers, M.-C. Kok-Escalle, op. cit. p. 45-49.

34. Horatius, De Arte poetica, 343 : «omne tulit punctum qui miscuit utile dulci » (la perfection, c'est de réunir l'utile et l'agréable).

35. Les dialogues 47 à 56 nous font suivre les différentes étapes d'un voyageur en France et le « Discours sur le véritable Etat de France [...]. En l'an $1673 »$ ne compte pas moins de vingt pages.

36. Ainsi Piélat insère dans ses dialogues de courtes phrases de ce type: « N'avez-vous point connu le docteur Patin?» (p. 37), « Connaissez-vous Monsieur le Maréchal de Turenne? » (p. 39).

37. Il s'agit de la guerre de Candie en Crète commencée en 1645 par le sultan Ibrãhim Ier. En 1669 , le gouverneur vénitien Morosini rend Candie aux Turcs.

38. Constituant un genre situé entre le fait divers et la nouvelle romanesque, les histoires connurent une très grande popularité aux XVIIe et XVIIIe siècles (M. Lever, Le roman français au XVIIe siècle, Paris P.U.F., 1981, p. 69).

39. Madeleine de Scudéry met en scène dans Le Grand Cyrus (10 vol., 1649-1653), la société précieuse de l'Hôtel de Rambouillet et de son propre salon.

40. Ch. Sorel (1599-1674) évoque dans l'Histoire comique de Francion (1622) des aventures grossières et bouffonnes dans des milieux louches de débauchés et de voleurs.

41. G. May, Le dilemne du roman au XVIIIe siècle, New Haven Yale University press, Paris P.U.F., 1963, 23-28.

42. M. van Strien-Chardonneau, « Le statut du français, langue seconde selon Isabelle de Charrière: langue de culture, langue utilitaire? " in M.-C. Kok-Escalle et F. Melka réd., Changements politiques et statut des langues. Histoire et épistémologie 1780-1945, Amsterdam, Rodopi, 2001, 41-52.

43. Il est à noter que dans certaines écoles françaises au XVIIe siècle, comme en Overijssel par exemple, les maîtres ou maîtresses sont tenus d'enseigner uniquement la langue française, soit pour ne pas faire concurrence au maître de langue hollandaise soit pour faciliter aux élèves l'apprentissage du français (Bijdragen tot de geschiedenis van Overijsel, Zwolle 1880, 48-59).

44. C'est nous qui soulignons.

45. Parival ajoute au Voyage de France un Voyage par la Hollande et les Provinces-Unies et même un Voyage d'Allemagne ou Des façons de faire des Universités d'Allemagne (dialogue 6 et 7). Baudet propose d'une section d'exercice à l'autre, sous une forme de feuilleton pour ainsi dire, les lettres d'un jeune voyageur signées Eugène (Exercices VI [1836] 7e éd. 1873 p. 181).

46. Parival, dialogues 6 et 7, 17 à 20; Marin, Abrégé de l'histoire de la Hollande; Cazelles, dialogues 18 à 27/26 (9e éd. 1809, 20e éd. 1854); Baudet, images de la Hollande, Exercices VI.

47. Les ouvrages de J.B. Lusson, Tableau chronologique \& historique de la république des Provinces Unies depuis les Cattes jusqu'á nos jours, Dordrecht P. van Braam, et A.M. Cerisier, Tableau de l'histoire générale des Provinces-Unies, 10 vol., Utrecht chez J. van Schoonhoven \& comp. 1777/1784, tous deux maîtres d'école française à Dordrecht, présentent des textes illustratifs des événements sélectionnés dans les chronologies; ils sont utilisés dans les écoles de Dordrecht, et celui de Lusson est même utilisé dans l'école avec pensionnat de jeunes filles de sa femme, comme en témoigne le Journal encyclopédique de 1776, cf. C. Esseboom, op.cit. p. 314 et 321. 


\section{RÉSUMÉS}

Aux Pays-Bas, l'enseignement du français a connu une institutionnalisation tardive avec la loi de 1863 pour le secondaire et celle de 1876 pour le supérieur. Mais dans les Provinces-Unies il y a une longue tradition du métier de maître de langues. Du XVII ${ }^{\mathrm{e}}$ au XIX ${ }^{\mathrm{e}}$ siècles, les maîtres, souvent plurilingues, ont réfléchi sur l'exercice de leur métier et ont produit des méthodes et autres ouvrages didactiques (Pielat, Mauger, du Casquet, Baudet, etc.). Le maître qui malgré les manuels est irremplaçable, doit être conscient des besoins de ses élèves; il s'interroge sur la qualité et l'efficacité de son enseignement. Par son enseignement de langue, il transmet un grand nombre de savoirs à l'élève et fonctionne finalement comme un médiateur social, éducatif et culturel. Tentant de joindre l'utile à l'agréable pour le profit des élèves, il leur fait découvrir la langue et la culture étrangères mais aussi leur propre monde. En ce sens il est un acteur de la formation identitaire de ses apprenants.

It was only in 1863 that French became part of the curriculum at Dutch secondary schools and in 1876 it received full status as a subject taught at university. However in Holland the teaching of French as a foreign language has a much longer tradition. From the seventeenth century onwards language teachers, often multi-lingual themselves, reflected on the practicalities of their speciality and produced a vast number of textbooks and other educational works (Pielat, Mauger, Du Casquet, Baudet etc.). The teacher, who in spite of his books remains essential, should be conscious of his pupils' needs, make sure of the quality of his teaching and achieve the desired results. Through his work as a teacher he confers to his pupil a vast amount of knowledge in many fields, so that in fact he also acts as a social, educational and cultural intermediary. In an effort to combine the useful and the pleasurable he enables his pupils not only to find out about a foreign language and its culture, but also their own world. Thus his action helps to shape the identity of those he teaches.

\section{INDEX}

Mots-clés : institutionnalisation, Maître de langues, médiateur éducatif et culturel, médiateur social, Pays-Bas, professeur, Provinces-Unies, XVIIe-XIXe siècles

Keywords : Dutch secondary schools, educational and cultural intermediary, Holland, Language schoolmaster, social intermediary, teacher, XVIth-XIXth centuries 